\title{
Erratum
}

\section{A Faithful Record of Stressful Life Events Preserved in the Dental Developmental Record of a Juvenile Gorilla ${ }^{1}$}

\author{
Gary T. Schwartz, ${ }^{2,6}$ Don J. Reid, ${ }^{3}$ M. Christopher Dean, ${ }^{4}$ \\ and Adrienne L. Zihlman ${ }^{5}$
}

Published Online September 7, 2006

Due to vendor errors in the correction process, the following errors were published in the online versions of this article.

The word "Recorded" in the title of the paper as originally published online should be "Preserved" as above.

\section{INTRODUCTION}

1. The third sentence of the first paragraph is missing the word "to" before "map" as part of the infinitive phrase. The sentence should begin: "New techniques have emerged to map the growth of individual teeth and entire dentitions..."

2. The first sentence of the fifth paragraph is missing the main verb "appear" after the word "develop." The sentence should begin:

\footnotetext{
${ }^{1}$ The online version of the original article can be found at http://dx.doi.org/10.1007/s10764-0069051-2.

${ }^{2}$ Department of Anthropology and Institute of Human Origins, Arizona State University, Tempe, Arizona.

${ }^{3}$ Department of Oral Biology, The Dental School, Newcastle upon Tyne, NE2 4BW, U.K.

${ }^{4}$ Evolutionary Anatomy Unit, Department of Anatomy and Developmental Biology,

University College, Gower Street, London WC1E 6BT, U.K.

${ }^{5}$ Department of Anthropology, University of California at Santa Cruz, California.

${ }^{6}$ To whom correspondence should be addressed; e-mail: garys.iho@asu.edu.
} 
"The incremental lines preserved as dental tissues develop appear in 2 forms ..."

\section{MATERIALS AND METHODS}

\section{Subject}

There is a superfluous period after the parenthetical phrase "( 3 yr 3 mo; 26.8)" at the beginning of the subsection. The subsection should begin with the following sentence, instead of the two sentence fragments as originally published online: "A healthy, captive juvenile western lowland gorilla (Gorilla gorilla gorilla; studbook no. 1268/650; studbook name OKLA $13 / \mathrm{G}$ ) died suddenly in an accident (AT 3 yr $3 \mathrm{mo} ; 26.8 \mathrm{~kg}$ ), not from a chronic or acute illness."

\section{Methods}

The first sentence of the second paragraph has a superfluous word "them" after the word hemimaxilla and should read: "We carefully extracted and cleaned each tooth from a hemimandible and hemimaxilla (Fig. 1c)."

\section{RESULTS}

\section{Age at Death, Crown Initiation, and Crown Formation Times}

All abbreviations that appear as "CFT" should read as "CFTs" throughout the subsection. 